\title{
Dispersion and Reinforcing Potential of Carboxymethylated Nanofibrillated Cellulose Powders Modified with 1-Hexanol in Extruded Poly(Lactic Acid) (PLA) Composites
}

\author{
C. Eyholzer $\cdot$ P. Tingaut $\cdot$ T. Zimmermann • \\ K. Oksman
}

Published online: 29 July 2012

(C) Springer Science+Business Media, LLC 2012

\begin{abstract}
Bionanocomposites of poly(lactic acid) (PLA) and chemically modified, nanofibrillated cellulose (NFC) powders were prepared by extrusion, followed by injection molding. The chemically modified NFC powders were prepared by carboxymethylation and mechanical disintegration of refined, bleached beech pulp (c-NFC), and subsequent esterification with 1-hexanol (c-NFC-hex). A solvent mix was then prepared by precipitating a suspension of c-NFC-hex and acetone-dissolved PLA in ice-cold isopropanol (c-NFC-hex ${ }_{\mathrm{sm}}$ ), extruded with PLA into pellets at different polymer/fiber ratios, and finally injection molded. Dynamic mechanical analysis and tensile tests were performed to study the reinforcing potential of dried and chemically modified NFC powders for PLA composite applications. The results showed a faint increase in modulus of elasticity of $10 \%$ for composites with a loading of $7.5 \%$ $\mathrm{w} / \mathrm{w}$ of fibrils, irrespective of the type of chemically modified NFC powder. The increase in stiffness was accompanied by a slight decrease in tensile strength for all samples, as compared with neat PLA. The viscoelastic properties of the composites were essentially identical to neat PLA. The absence of a clear reinforcement of the polymer matrix was attributed to poor interactions with PLA and insufficient dispersion of the chemically modified NFC powders in the composite, as observed from scanning electron microscope images. Further explanation was found in the decrease of the
\end{abstract}

C. Eyholzer · P. Tingaut $\cdot$ T. Zimmermann $(\bowtie)$

Applied Wood Materials Laboratory, Empa, Swiss Federal

Laboratories for Materials Science and Technology,

Dübendorf, Switzerland

e-mail: Tanja.Zimmermann@empa.ch

C. Eyholzer · K. Oksman

Division of Materials Science, Luleå University of Technology,

Luleå, Sweden thermal stability and crystallinity of the cellulose upon carboxymethylation.

Keywords Nanofibrillated cellulose $\cdot$ Polylactic acid · Esterification $\cdot$ Extrusion $\cdot$ Bio-nanocomposites

\section{Introduction}

Polylactic acid (PLA) is an aliphatic polyester with high strength and stiffness that can be made from annually renewable resources, like starch from corn or potato, and sugar from cane or beet. Being a thermoplastic, it is easily processed on standard plastic equipment to yield molded parts, films or fibers. Due to its biocompatibility and biodegradability, PLA is suitable for medical devices or for use in the industrial packaging field [1, 2].

The isolation and use of cellulose nanostructures and their application in composite materials has gained increasing attention due to their inherent properties like high strength and stiffness combined with low weight, biodegradability and renewability [3]. Their beneficial mechanical properties arise from $\beta-1,4$ linked glucopyranose chains, aligned into highly ordered (crystalline) domains by intra- and intermolecular hydrogen bonds. These crystallites are linked by amorphous domains to form bundles of fibrils [4]. Over the last years, two categories of nanocelluloses have been mainly used in order to reinforce a PLA matrix: cellulose nanowhiskers (CNW) and nanofibrillated cellulose (NFC). CNW are mostly obtained by acid hydrolysis of cellulose pulp from wood, or other sources of cellulose, such as algae, bacteria, tunicates or wheat. The chemical treatment dissolves the amorphous fraction of cellulose and needle shaped crystallites are isolated [5-7]. Mechanical isolation of pulp (usually applied in combination with a chemical, mechanical 
or enzymatic pretreatment) results in breakup of the cellulose fibers and yields semi-crystalline fibrils and fibril bundles with diameters in the range between 3.5 and $100 \mathrm{~nm}$, called NFC [8]. Due to their beneficial mechanical properties and their inherent biodegradability and biocompatibility, CNW and NFC are qualified for the development of nanocomposites with PLA. However, due to their large, hydrophilic surface area $[4,8]$, these nanomaterials tend to undergo agglomeration when compounding with hydrophobic polymers. In response, several strategies have been examined to overcome this issue in composites with PLA and the resulted mechanical properties are listed in Table 1.

In first experiments, slurries containing $\mathrm{CNW}$ in $\mathrm{N}, \mathrm{N}$-dimethyl acetamide/lithium-chloride (DMAc/LiCl) and a maleic anhydride coupling agent were directly fed into a PLA melt during extrusion. The results indicated that the CNW had a positive effect on all mechanical properties (stiffness, strength and strain) of the composites, even though the additives showed tendency for agglomeration [9]. In a subsequent study, aqueous suspensions of CNW or NFC in combination with polyethylene glycol (PEG) were fed into the PLA melt. Again, the formation of agglomerates could not be avoided [10]. Nanocomposites were also prepared by solution casting. CNW were freeze-dried from an aqueous suspension, after solvent exchange to tert-butanol and after modification with a surfactant. The powders were then redispersed in chloroform and the suspensions were mixed with dissolved PLA, followed by evaporation of the solvent. The surfactant considerably improved dispersion of the CNW in the PLA matrix compared to the unmodified CNW, as was observed by transmission electron microscopy (TEM). However, agglomeration of CNW was not completely prevented. In addition, the surfactant clearly decreased the storage modulus of the PLA matrix [11]. Similar results were obtained when surfactant modified, freeze-dried CNW were extruded with PLA. Even though, the surfactant greatly improved the dispersion of CNW in the PLA matrix (compared to unmodified CNW), the composites did suffer from a slight decrease in stiffness and strength, caused by the plasticizing effect of the surfactant $[12,13]$. Extrusion of PLA with a masterbatch of CNW and polyvinyl alcohol $(\mathrm{PVOH})$ (directly fed to the melt as solution or as a solid mixture after freeze-drying) resulted in phase separation of the immiscible polymers. The CNW were located predominantly in the discontinuous PVOH phase and therefore only a limited increase in tensile modulus and strength of PLA was observed [12]. Later, nanocomposites were prepared by gradually adding PLA to a thoroughly dispersed suspension of unmodified NFC in acetone, followed by solution casting. The films were then melt-kneaded in a rotary roller mixer and hot-pressed. This procedure seems somewhat irritating, as unmodified NFC is expected to show instantaneous sedimentation in the described 9:1 w/w acetone/water mixture. Nevertheless, microscopic images showed a homogeneous distribution of NFC in the fully amorphous PLA matrix and tensile tests showed an increase of 25 and $16 \%$ for modulus and tensile strength, respectively [14]. A very similar procedure was then applied to reinforce a semi-crystalline PLA with unmodified NFC. The thin film composites were either quenched in liquid nitrogen or annealed in a hot press at $100{ }^{\circ} \mathrm{C}$ to obtain fully amorphous or crystallized composites, respectively. The tensile moduli and strengths of the amorphous composites were approximately in the same range as those of the precedent experiment, while the crystallized samples showed slightly higher values. Dynamic mechanical analysis (DMA) showed a clear increase in storage modulus above the glass transition for composites containing unmodified NFC compared to neat PLA, especially for

Table 1 Overview on modulus, tensile strength and elongation to break of selected composites containing NFC or whiskers in PLA

\begin{tabular}{|c|c|c|c|c|c|c|c|c|}
\hline \multirow[t]{2}{*}{ Source } & \multirow[t]{2}{*}{ Filler } & \multirow[t]{2}{*}{ Additives } & \multicolumn{2}{|c|}{ Modulus (GPa) } & \multicolumn{2}{|c|}{ Strength $(\mathrm{MPa})$} & \multicolumn{2}{|c|}{ Elongation to break $(\%)$} \\
\hline & & & Neat PLA & Composite & Neat PLA & Composite & Neat PLA & Composite \\
\hline Oksman et al. [9] & Whiskers & DMAc/LiCl PLA-MA & $2.9 \pm 0.1^{\mathrm{a}}$ & $3.9 \pm 0.3$ & $40.9 \pm 3.2^{\mathrm{a}}$ & $77.9 \pm 6.7$ & $1.9 \pm 0.2^{\mathrm{a}}$ & $2.7 \pm 0.5$ \\
\hline \multirow[t]{2}{*}{ Mathew et al. [10] } & NFC & PEG & $2.0 \pm 0.2$ & 2.3 & $58 \pm 6$ & $59 \pm 2$ & $4.2 \pm 0.6$ & $3.3 \pm 0.2$ \\
\hline & Whiskers & PEG & & 2.1 & & $47 \pm 5$ & & $5.4 \pm 1.8$ \\
\hline Bondeson et al. [12] & Whiskers & Beycostat surfactant & $2.7 \pm 0.1$ & $3.1 \pm 0.2$ & $62.8 \pm 1.0$ & $52.4 \pm 0.4$ & $19.5 \pm 9.7$ & $3.1 \pm 0.2$ \\
\hline Bondeson et al. [13] & Whiskers & $\mathrm{PVOH}$ & $3.3 \pm 0.1$ & $3.6 \pm 0.3$ & $71.9 \pm 2.0$ & $67.7 \pm 0.8$ & $3.4 \pm 0.2$ & $2.4 \pm 0.2$ \\
\hline Iwatake et al. [14] & NFC & - & 3.4 & 4.3 & 56.2 & 66.0 & N.a. & N.a. \\
\hline \multirow[t]{2}{*}{ Suryanegara et al. [15] } & NFC & - & $3.3 \pm 0.2^{\mathrm{b}}$ & $3.9 \pm 0.1^{\mathrm{b}}$ & $57.7 \pm 1.5^{\mathrm{b}}$ & $63.4 \pm 1.1^{\mathrm{b}}$ & $6.8 \pm 2.1^{\mathrm{b}}$ & $2.5 \pm 0.2^{\mathrm{b}}$ \\
\hline & & - & $4.0 \pm 0.1$ & $4.6 \pm 0.1$ & $60.9 \pm 1.6$ & $64.4 \pm 0.8$ & $3.1 \pm 0.4$ & $2.0 \pm 0.1$ \\
\hline Jonoobi et al. [17] & NFC & - & $2.9 \pm 0.6$ & $3.6 \pm 0.7$ & $58.9 \pm 0.5$ & $71.2 \pm 0.6$ & $3.4 \pm 0.4$ & $2.7 \pm 0.1$ \\
\hline \multicolumn{2}{|l|}{ c-NFC-hex sm $5 \%$ w/w } & - & $3.6 \pm 0.1$ & $3.7 \pm 0.1$ & $66.2 \pm 1.6$ & $59.6 \pm 0.8$ & $4.1 \pm 1.3$ & $3.3 \pm 0.6$ \\
\hline
\end{tabular}

The loading of nanoparticles in each composite was $5 \% \mathrm{w} / \mathrm{w}$

${ }^{a}$ Values for PLA extruded with indicated additives

b Values for purely amorphous PLA 
crystallized samples [15]. An increase in storage modulus above the glass transition was also found for composites obtained by solution casting of a mixture of acetylated NFC and PLA from chloroform. Interestingly, for the same fibril loadings, the acetylated NFC showed a lower reinforcement in the rubbery plateau than unmodified NFC, which was attributed to a reduced number of hydrogen bonds that could be formed due to the acetyl groups [16]. In a most recent study, unmodified NFC was solvent exchanged from water to acetone before mixing with dissolved PLA. Again, the mixture was evaporated and dried before extrusion. An increase in modulus of $25 \%$ and strength of $20 \%$ was observed. However, scanning electron images still proved the formation of agglomerates within the composites [17].

Over all as shown in Table 1, the reinforcement of PLA with CNW and NFC led to a maximum increase in stiffness of 42 and $25 \%$, respectively. Most of the studies did not show any improvement in strength, which was mainly attributed to the poor filler dispersion and interaction between the nanofibers and the PLA. Interestingly, the highest increase in strength and strain reported so far has been measured for unmodified CNW and NFC $[9,17]$.

It is therefore not surprising that in all composites containing PLA presented so far, at least to a certain extent, NFC agglomeration was observed. However, as generally emphasized, perfect dispersion in the matrix to yield a large interfacial area and strong interaction between the components is crucial for exploiting the full reinforcing potential. In addition, the proposed preparation procedures have involved either a liquid feeding of the components to the extruder or a solution casting step.

Therefore, a simple procedure for the development of nanocomposites containing perfectly dispersed NFC or CNW (provided in dry form) in PLA, allowing the scale up to industrially competitive processes, has not been presented so far. We showed in an earlier study [18] that carboxymethylated NFC (c-NFC) powder can be redispersed in water after drying and therefore, it is interesting to see if this cellulose type can be even redispersed in a non-polar polymer during the extrusion process. Accordingly in this study, c-NFC in dry powder form was used as starting material and its carboxymethyl groups were esterified with 1-hexanol in order to increase the compatibility at the fiber-polymer matrix interface. Nanocomposites were prepared by compounding extrusion and the test samples were injection molded. The fractured surfaces of the tensile tested samples were analyzed by scanning electron microscopy (SEM) to study the dispersion of the NFC in PLA. The mechanical properties of the nanocomposites were analyzed by tensile tests, dynamic mechanical analysis (DMA) and melt shear tests and compared to neat PLA samples.

\section{Materials and Methods}

Materials

Refined, bleached beech pulp (RBP) was provided by J. Rettenmaier \& Söhne GmbH (Rosenberg, Germany) as an aqueous suspension (Arbocel B1011). The dry material content of the suspension was measured by gravimetry to $12.4 \%$ w/w. The Kappa number and the hemicellulose content were 1.0 and $7.07 \%$, respectively, as determined by chemical analysis. Mono-chloroacetic acid (sodium salt, purity $\geq 98 \%, \quad \mathrm{M}=116.48 \mathrm{~g} / \mathrm{mol}$ ), 1 -hexanol (purity $\geq 98 \%, \mathrm{M}=102.17 \mathrm{~g} / \mathrm{mol})$ and sulfuric acid $\left(\mathrm{H}_{2} \mathrm{SO}_{4}\right.$, p.a. 95-97 \%, M = 98.08 g/mol) were purchased from Merck. Sodium hydroxide $(\mathrm{NaOH}$, purity $\geq 98 \%, \mathrm{M}=40.0 \mathrm{~g} / \mathrm{mol})$ and dichloromethane $\left(\mathrm{CH}_{2} \mathrm{Cl}_{2}\right.$, purity $\geq 98 \%, \mathrm{M}=84.93 \mathrm{~g} /$ mol) were purchased from Fluka and Carl Roth GmbH. Polylactic acid (PLA, 2002 D grade, Nature Works ${ }^{\text {TM }}$ ) was provided by Cargill Dow LCC (Minnetonka, MN, USA).

\section{Sample Preparation}

\section{Synthesis of Carboxymethylated NFC (c-NFC)}

Carboxymethylation of RBP was performed following an earlier protocol [18] with slight modifications: $806 \mathrm{~g}$ of RBP (corresponding to $100 \mathrm{~g}$ of dry cellulose) was dispersed in $4.5 \mathrm{~L}$ of a $3: 5 \mathrm{v} / \mathrm{v}$ ethanol/isopropanol mixture in a $10 \mathrm{~L}$ glass reactor. After a swelling time of 4 days, the RBP was activated by dropping $493 \mathrm{~g}$ of a $5 \% \mathrm{w} / \mathrm{w}$ aqueous $\mathrm{NaOH}$ into the mixture. $53.9 \mathrm{~g}$ of monochloroacetic acid were added. The temperature was set to $60{ }^{\circ} \mathrm{C}$ and the reaction conducted for $2 \mathrm{~h}$. (Fig. 1). The resulting suspension was washed with a $1 / 1 \mathrm{v} / \mathrm{v}$ mixture of a $0.05 \mathrm{M}$ aqueous acetic acid solution and a $5 / 3 \mathrm{v} / \mathrm{v}$ isopropanol/ethanol mixture, and finally dispersed in $9.5 \mathrm{~L}$ of a $0.01 \mathrm{M} \mathrm{NaOH}$. Mechanical disintegration was done using a high-shear homogenizer (Microfluidizer type M-110Y, Microfluidics Corporation, USA). Two chamber setups were used. The suspensions were pumped 3 times through $\mathrm{H} 230 \mathrm{Z}_{400 \mu \mathrm{m}}$ and $\mathrm{H} 30 \mathrm{Z}_{200 \mu \mathrm{m}}$ chambers, followed by another 3 passes through $\mathrm{H} 30 \mathrm{Z}_{200 \mu \mathrm{m}}$ and $\mathrm{F}_{2} 0 \mathrm{Y}_{75 \mu \mathrm{m}}$ chambers. The c-NFC suspension was then solvent-exchanged to isopropanol (centrifugation at $5,000 \mathrm{rpm}$ for $15 \mathrm{~min}$, followed by discarding the supernatant and dispersion of the c-NFC sediment in isopropanol; procedure was repeated three times). The c-NFC was finally dried from isopropanol at $60^{\circ} \mathrm{C}$ under stirring to avoid hornification (irreversible agglomeration) [18]. A degree of substitution (DS) of 0.23 was determined by conductometric titration (average of three measurements). 
Fig. 1 Synthesis of c-NFC (center) and c-NFC hexanoate (right). The DS of functional groups $\mathrm{R}$ and $\mathrm{R}$ ' were determined by conductometric titration: $D S_{\mathrm{COOH}}=0.09$ and $D S_{\text {hex }}=0.14$

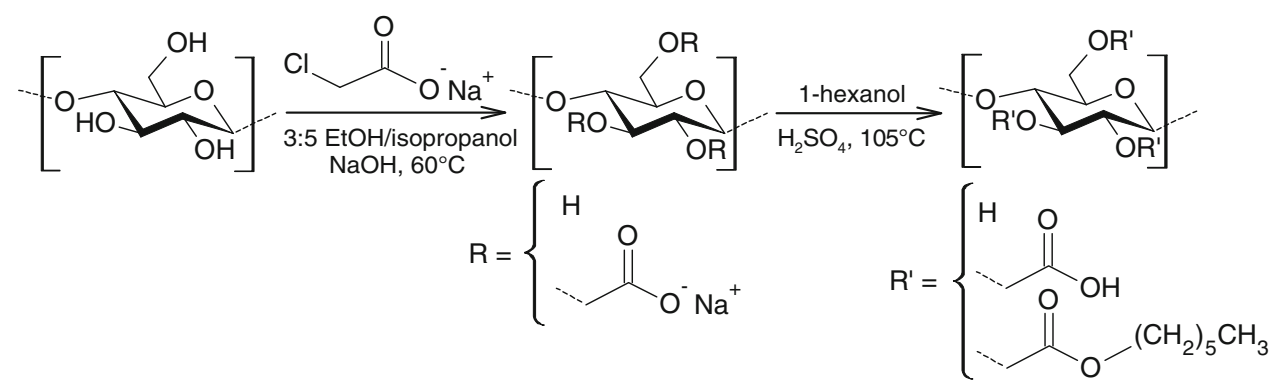

Synthesis of c-NFC Hexanoate (c-NFC-hex)

$40 \mathrm{~g}$ of dried c-NFC was dispersed in $1.0 \mathrm{~L}$ of 1-hexanol using a high-shear mixer ( $\mathrm{T} 25$ basic, IKA-Werke, Stauffen, Germany) in a $2 \mathrm{~L}$ glass reactor, equipped with a mechanical stirrer and reflux cooler. $2 \mathrm{~mL}$ of $\mathrm{H}_{2} \mathrm{SO}_{4}$ was added with a pipette. The reaction was conducted at $105{ }^{\circ} \mathrm{C}$ for $72 \mathrm{~h}$ (Fig. 1). The suspension was centrifuged at $5,000 \mathrm{rpm}$ for $30 \mathrm{~min}$. The sediment was washed twice with $700 \mathrm{~mL}$ of a $1 / 1 \mathrm{v} / \mathrm{v}$ acetone $/ 0.001 \mathrm{M} \mathrm{NaOH}$ mixture and 3 times with pure acetone. Then, the c-NFC-hex was dried at $60{ }^{\circ} \mathrm{C}$ in an oven under frequent manual stirring.

The DS of the unreacted carboxymethyl groups was determined, using Eq. 1:

$$
\begin{aligned}
& D S_{\mathrm{COOH}}=\frac{n_{\mathrm{COOH}}}{n_{\mathrm{AGU}}} \\
& =n_{\mathrm{COOH}}\left(\frac{m_{0}+\left(n_{\mathrm{COOH}} \cdot M_{h e x}\right)}{M_{A G U}+\left(D S_{c-N F C} \cdot M_{h e x}\right)+\left(D S_{c-N F C} \cdot M_{c m}\right)}\right)^{-1}
\end{aligned}
$$

with $n_{\mathrm{COOH}}$ and $n_{A G U}$ being the amount of free $\mathrm{COOH}$ groups (measured by conductometric titration), and the amount of anhydroglucose units, respectively. $m_{0}$ is the mass of the weighted sample of dry c-NFC hexanoate. $M_{h e x}, M_{A G U}$ and $M_{c m}$ denote the molecular masses of the hexanoate group $\left(\left(\mathrm{CH}_{2}\right)_{5} \mathrm{CH}_{3}, 85 \mathrm{~g} / \mathrm{mol}\right)$, the anhydroglucose unit $(162.14 \mathrm{~g} / \mathrm{mol})$ and the carboxymethyl group $(57 \mathrm{~g} / \mathrm{mol})$. $D S_{c-N F C}$ denotes the DS of the c-NFC intermediate product (0.23). The final DS of free carboxymethyl groups and hexanoate groups were calculated using Eq. 2 to $D S_{\mathrm{COOH}}=0.09$ and $D S_{\text {hex }}=0.14$, respectively:

$D S_{\text {hex }}=D S_{c-N F C}-D S_{C O O H}$

\section{Preparation of Solvent Mix PLA (c-NFC-hex $\left.{ }_{s m}\right)$}

$16.0 \mathrm{~g}$ of c-NFC-hex was dispersed in $400 \mathrm{ml}$ of $\mathrm{CH}_{2} \mathrm{Cl}_{2}$ using a high-shear mixer. In a separate flask, $16.0 \mathrm{~g}$ of PLA was dissolved in $400 \mathrm{~mL}$ of acetone overnight. The mixtures were combined in a $1 \mathrm{~L}$ flask and homogenized using a high-shear mixer. $\mathrm{CH}_{2} \mathrm{Cl}_{2}$ was distilled off the suspension using a Rotavap and the remaining suspension was dropped into $2 \mathrm{~L}$ of ice-cold isopropanol. The precipitate was dried in an oven at $60{ }^{\circ} \mathrm{C}$.

\section{Compounding Extrusion}

All materials were dried prior to extrusion at $50{ }^{\circ} \mathrm{C}$ overnight. A MEGALab 18 co-rotating twin-screw extruder (Coperion W\&P, Stuttgart, Germany) containing 7 heating zones (set to $165 ; 170 ; 170 ; 180 ; 180 ; 190$ and $200{ }^{\circ} \mathrm{C}$, respectively) was used. The screw speed was set to $150 \mathrm{rpm}$.

In the first step, 3 different material combinations were prepared to make extruded masterbatches: 10/90 w/w c-NFC/PLA; 10/90 w/w c-NFC-hex/PLA and 10/90 w/w c-NFC-hex ${ }_{s m} /$ PLA. Neat PLA was subjected to the same process to ensure similar processing history. All extrudates were pelletized.

In the second step, the extruded masterbatch pellets were diluted with fresh PLA in order to obtain mixtures containing a total of 2.5, 5.0 and 7.5\% w/w of dry material (c-NFC/c-NFC-hex and c-NFC-hex ${ }_{s m}$, respectively). The mixtures were extruded using the same parameters and subsequently pelletized.

\section{Injection Molding}

The prepared composites and the PLA were injection molded using a Haake MiniJet laboratory injection molding machine (Thermo Scientific, Karlsruhe, Germany). Dog-bone specimens according to ASTM D638-2003 standard, type 5 (thickness $3.3 \mathrm{~mm}$, width $3.2 \mathrm{~mm}$, length $60 \mathrm{~mm}$, parallel length $1_{0} 20 \mathrm{~mm}$ ) and flat discs (diameter $32 \mathrm{~mm}$, thickness $1.6 \mathrm{~mm}$ ) were injection molded. The following parameters for dog-bone specimens and flat discs (in brackets) were used: cylinder temperature $200{ }^{\circ} \mathrm{C}$ $\left(200{ }^{\circ} \mathrm{C}\right)$, mold temperature $60^{\circ} \mathrm{C}\left(80{ }^{\circ} \mathrm{C}\right)$, injection pressure 500 bar (600 bar), injection time $30 \mathrm{~s}$ (30 s), hold pressure 100 bar (100 bar), hold time $60 \mathrm{~s}$ (60 s). 
Measurements

\section{Tensile Tests}

Modulus of elasticity (MOE) and nominal tensile strength were determined according to EN ISO 527-1:1996 with slight modifications. A Universal Testing System (Zwick 1474, Ulm, Germany), equipped with a $20 \mathrm{kN}$ load cell was used. The samples were conditioned for 1 week at $35 \%$ relative humidity and $20^{\circ} \mathrm{C}$. Elongation of the samples was measured by mechanical strain detection. MOE values were determined by the slope of the linear interpolation line of the curves between 0.1 and $0.3 \%$ strain. The initial cross head speed was set to $1 \mathrm{~mm} / \mathrm{min}$ and increased to $2 \mathrm{~mm} / \mathrm{min}$ after the determination of the MOE to reduce creeping of the samples. For each sample, 10 replicates were measured.

\section{Dynamic Mechanical Analysis (DMA)}

Viscoelastic properties of injection molded samples were studied by using a RS IIIa Rheometrics System Analyzer (TA Instruments, Delaware, USA) in three point bending mode. The composite flat discs were cut into rectangular specimens with $10.0 \mathrm{~mm}$ width and $40.0 \mathrm{~mm}$ length and were conditioned for 1 week at $20{ }^{\circ} \mathrm{C}$ and $35 \%$ relative humidity.

Dynamic heating scans were performed from 30 to $140{ }^{\circ} \mathrm{C}$ at a heating rate of $2{ }^{\circ} \mathrm{C} / \mathrm{min}$ with an initial static force of $75 \mathrm{~g}$. The heating scans were followed by $2 \mathrm{~min}$ of isothermal equilibration at $140{ }^{\circ} \mathrm{C}$ and dynamic cooling scans at a cooling rate of $2{ }^{\circ} \mathrm{C} / \mathrm{min}$ with an initial static force of $2 \mathrm{~g}$. The static force in all scans was set $15 \%$ higher than the dynamic force. Initial load strain and upper limit for the applied load strain were set to 0.05 and $0.1 \%$, respectively. All measurements were done at a frequency of $1 \mathrm{~Hz}$. Purge gas was dry air over the whole temperature range.

\section{Melt Flow Index (MFI)}

Melt flow indexes (MFI) of neat PLA and composites were measured using a MI-1 apparatus (Goettfert, Buchen, Germany). The specimens were melted at $190{ }^{\circ} \mathrm{C}$ for $2 \mathrm{~min}$. The mass of the piston was $2.16 \mathrm{~kg}$. The mass of the polymer composites that flowed through the capillary was measured after $30 \mathrm{~s}$. An average of 5 measurements was calculated for each sample.

\section{Shear Tests}

The viscoelastic properties of the injection molded flat disc samples in shear (storage modulus G', loss modulus G" and viscosity $\gamma$ ) were measured using an ARES, Rheometric Scientific Inc. (New Jersey, USA) in parallel plate geometry (diameter $50 \mathrm{~mm}$ ). Dynamic frequency sweep tests were performed at 180 and at $200{ }^{\circ} \mathrm{C}$. The flat discs were melted and compressed until fully occupying the area of the plates (disc gap between 0.8 and $1.2 \mathrm{~mm}$ ). A delay of $180 \mathrm{~s}$ after the preload ensured complete relaxation of the samples before the tests. A shear strain of $1.0 \%$ with frequencies from 0.08 to $80 \mathrm{~Hz}(0.50265-502.65 \mathrm{rad} / \mathrm{s})$ was applied and 9 points per decade were recorded. Two specimens per sample were measured and the values were averaged.

\section{Scanning Electron Microscopy (SEM)}

Fractured dog-bone tensile specimens were cut with a razor blade to obtain cubes of $3 \mathrm{~mm}$ edge length. The cubes were mounted on a sample holder. The fractured surfaces were sputter coated with a platinum layer of $7.0 \mathrm{~nm}$ (BAL-TEC MED 020 Modular High Vacuum Coating System with integrated quartz crystal film thickness and coating rate measuring, BAL-TEC AG, Liechtenstein) in Ar as a carrier gas at $5 \times 9 \times 10^{-2}$ mbar. Images were recorded in a FEI NovaNanoSEM (FEI Company, Hillsboro, OR, USA), equipped with a Schottky field emission gun. The following parameters were used: acceleration voltage of $5.0 \mathrm{kV}$ and working distance of $5.0 \mathrm{~mm}$. Of each sample, two specimens were examined.

\section{Results and Discussion}

\section{Materials Preparation}

Figure 1 presents the synthesis of c-NFC hexanoate. We started with a carboxymethyl cellulose (c-NFC) powder with a DS a 0.23 . The esterification reaction of c-NFC was performed after redispersion of the powder in 1-hexanol. After reaction and proper washing procedures, a DS of 0.14 was determined for c-NFC-hex, therefore confirming that more than $60 \%$ of the original carboxyl acid functions were esterified.

After the reaction, when left without stirring, the c-NFC-hex in the mixture sedimented within a couple of minutes. During washing of c-NFC-hex with $1 / 1 \mathrm{v} / \mathrm{v}$ acetone $/ 0.001 \mathrm{M} \mathrm{NaOH}$, foam generation was observed, which could be the result of the formation of micellar structures of the alkyl side chains in aqueous medium. In pure acetone, c-NFC-hex did not form a stable suspension but sedimented after some minutes. The tendency towards sedimentation suggests the presence of agglomerates of nanofibrillated cellulose and must be kept in mind when discussing the results of the following measurements. 


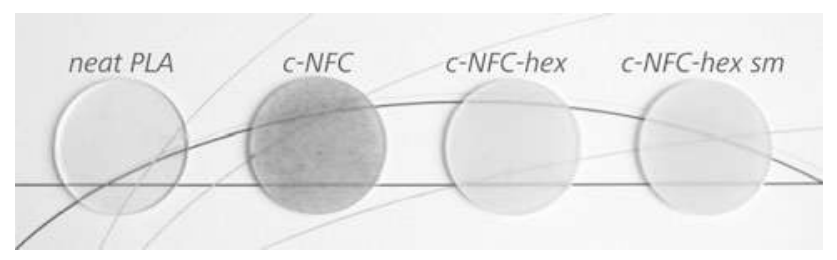

Fig. 2 Photographs of flat discs (thickness $1.6 \mathrm{~mm}$ ) of neat PLA and composite samples containing $5.0 \% \mathrm{w} / \mathrm{w}$ of fibrils. Dark coloration was attributed to thermal degradation of the matrix and fibrils

The various products (c-NFC, c-NFC-hex powder and c-NFC-hex ${ }_{s m}$ ) were extruded to yield three different composites, each with 3 loadings of fibrils, i.e. 2.5, 5.0 and 7.5\% w/w of fibrils. The extrudates were cut to pellets and injection molded to yield dog-bone shaped tensile testing samples and flat disc samples for DMA and melt shear measurements. While neat PLA samples were almost colorless and transparent, the composite samples showed the formation of a yellowish color (Fig. 2). This coloration was faint for composites containing c-NFC-hex ${ }_{s m}$ and c-NFC-hex but clearly more pronounced for composites containing c-NFC (almost dark brown color). In addition, the transparency of the samples was also reduced in the same order and in some samples agglomerates, big enough to be detected by naked eye, were observed.

\section{Characterization of Materials}

Figure 3 (left) shows the modulus of elasticity (MOE) of neat PLA (white column) and the composites containing c-NFC, c-NFC-hex and c-NFC-hex ${ }_{s m}$ (light, medium and dark gray columns, respectively), obtained from tensile tests. As it can be seen, only small differences between the MOE values of the different samples were measured. At the highest loading of $7.5 \% \mathrm{w} / \mathrm{w}, \mathrm{MOE}$ values increased

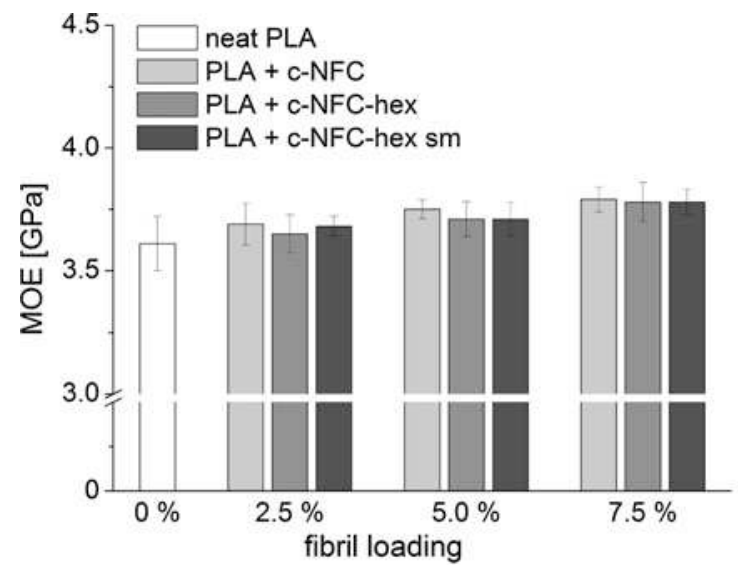

Fig. 3 Modulus of elasticity (MOE) (left) and tensile strength (right) for neat PLA and composites containing c-NFC, c-NFC-hex or $\mathrm{c}-\mathrm{NFC}-\mathrm{hex}_{\mathrm{sm}}$ in dependence of fibril loadings in $\mathrm{w} / \mathrm{w} \%$, obtained only $10 \%$ compared to neat PLA showing a modulus of 3.61 GPa, while for lower loadings the effect was even less pronounced. No differences in modulus were observed for composites containing different types of fibrils. Similar behavior was observed when analyzing the tensile strength of the samples (Fig. 3, right). Neat PLA showed a tensile strength of about $66 \mathrm{MPa}$ and again, the values of the composites showed little differences. The data suggests that with increasing loading of the fibrils the tensile strength even decreased, which was most pronounced for the composite containing c-NFC. From these results we must deduce that the reinforcing potential of the fibrils was not exploited and the fibrils acted as filler rather than as a reinforcing agent.

Nevertheless, moduli and tensile strengths measured for the developed composites are in the same range as the mechanical properties for composites containing $5 \% \mathrm{w} / \mathrm{w}$ of $\mathrm{NFC}$ or $\mathrm{CNW}$ in earlier reports (Table 1). It must however be considered that the values reported in this table have to be taken with care, as they are affected by the chosen test parameters (test method, sample dimensions, load cell, cross-head speed, etc.).

The results from tensile tests were further confirmed by DMA experiments. Figure $4 \mathrm{a}$ shows heating scans of the neat PLA (green dots) and the composites containing $7.5 \%$ w/w of c-NFC, c-NFC-hex and c-NFC-hex ${ }_{s m}$ (purple, olive and yellow dots, respectively). Neat PLA is in a glassy state at room temperature, with a storage modulus (top) of approximately $3 \mathrm{GPa}$. Upon heating, the $\tan \delta$ curve (bottom) peaked at approximately $60^{\circ} \mathrm{C}$, indicating the transition from a glassy to a rubbery state. The transition was followed by a short rubbery plateau of storage modulus up to $85^{\circ} \mathrm{C}$. Above this temperature, a faint drop in storage modulus was observed for neat PLA. This drop was attributed to the relaxation of molded-in stresses from

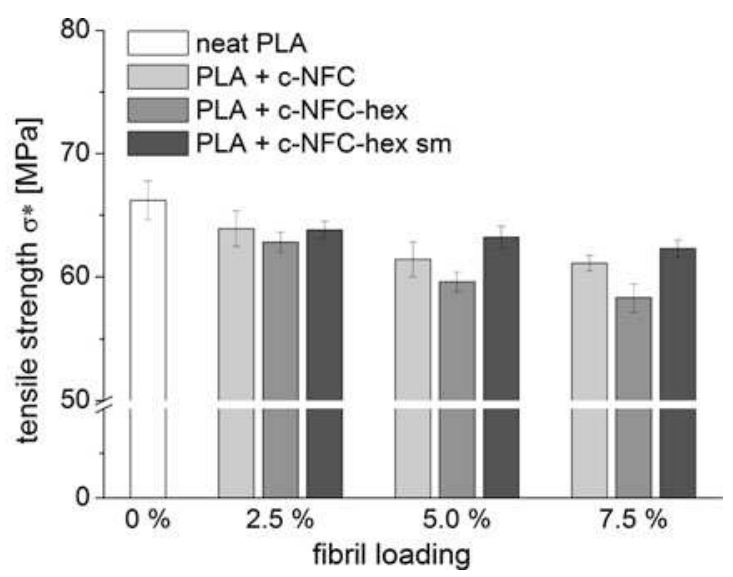

from tensile testing experiments. A linear scale was chosen to visualize the small differences in MOE between samples 

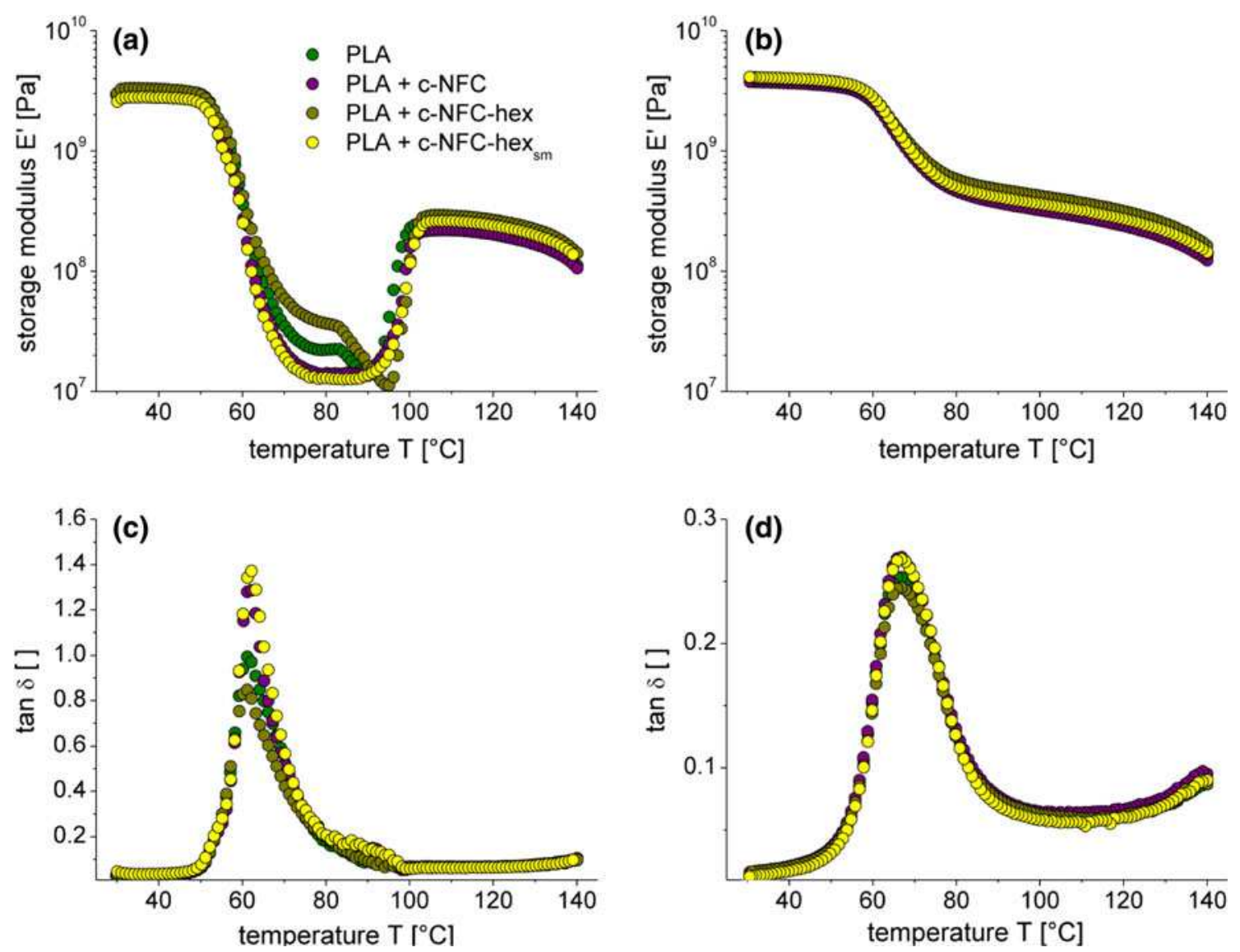

Fig. 4 Dynamic mechanical analysis (DMA) test heating scan (left) and cooling scan (right) of neat PLA and composites containing $7.5 \% \mathrm{w} / \mathrm{w}$ of c-NFC, c-NFC-hex or c-NFC-hex ${ }_{\mathrm{sm}}$ in three-point

bending geometry. The curves show the storage modulus E' $(\mathbf{a}, \mathbf{b})$ and $\tan \delta(\mathbf{c}, \mathbf{d})$ in dependence of temperature, recorded at a frequency $\omega$ of $1 \mathrm{~Hz}$. For better visualization, the $\tan \delta$ scale in (d) was enlarged

injection molding. Above $90{ }^{\circ} \mathrm{C}$, the storage modulus showed an increase of more than one decade, indicative for a crystallization process of the semicrystalline polymer matrix. At $100{ }^{\circ} \mathrm{C}$, a second, broad, plateau was reached, which slowly declined at higher temperatures approaching the melting temperature. The peak position and form was similar for all tested materials, only the peak height changed, which is indicative for the amount of molecules participating in the relaxation. Nevertheless, the fact that the peak position did not change can be interpreted as lack of interaction between the NFC and PLA.

The storage moduli of the composites showed basically the same temperature dependence as neat PLA. Minor differences were observed in the relaxation of the moldedin stresses, indicating slightly different cooling profiles (as samples were ejected manually from the mold, this could not be avoided completely).

Figure $4 \mathrm{~b}$ shows the subsequent cooling scan of neat PLA (green dots) and composites with $7.5 \%$ w/w NFC (purple, olive and yellow dots, respectively). A continuous rubbery plateau was found for all curves with a slight

increase in storage moduli towards lower temperatures. Upon further cooling, the samples changed into a glassy state, indicated by the $\tan \delta$ peak around $60^{\circ} \mathrm{C}$. Clearly, all the samples showed almost the same values in both scans, suggesting very similar viscoelastic properties in the examined temperature range. As already observed for the tensile tests, the results obtained with DMA confirmed that the fibrils did not lead to a significant increase in stiffness of the composites, and no evidence was seen on restriction of molecular movement of the PLA which is also indicative for poor interaction between the PLA and the nanofibers, chemically modified or not.

In addition to the measurements done at the glassy and rubbery states of PLA, the composites were also analyzed in their melted form with the aim to indirectly get information about the dispersion quality of the nanofibers inside the polymeric matrix, as the melt flow is expected to decrease with the addition of nanofibers and their dispersion. Figure 5 shows the melt flow index (MFI) of neat PLA and the composites in dependence of the type and loading of fibrils. For neat PLA (white column), an MFI of 


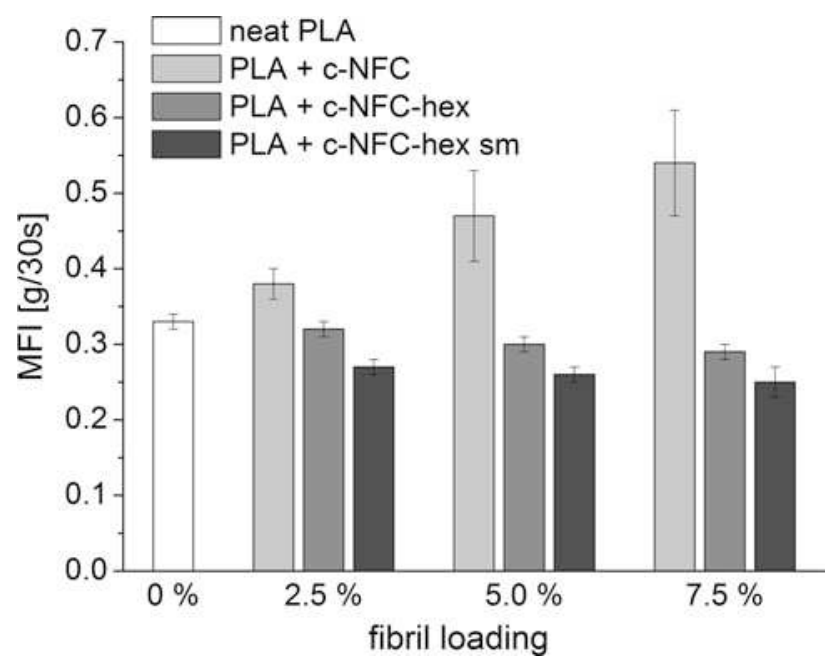

Fig. 5 Melt flow index (MFI) for neat PLA and composites containing c-NFC, c-NFC-hex or c-NFC-hex sm in dependence of fibril loadings in $\% \mathrm{w} / \mathrm{w}$
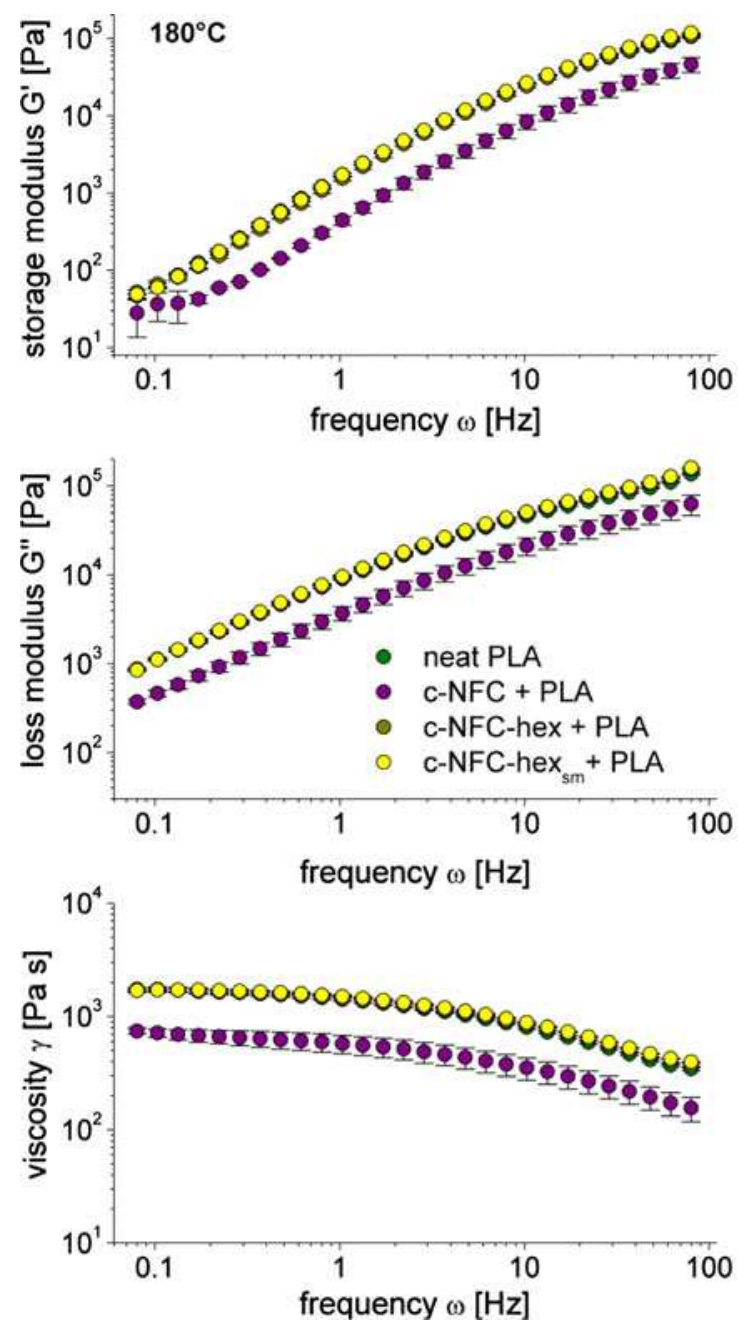

Fig. 6 Dynamic mechanical analysis (DMA) melt shear test in parallel plate geometry of neat PLA and composites containing $7.5 \%$ $\mathrm{w} / \mathrm{w}$ of c-NFC, c-NFC-hex or c-NFC-hex $\mathrm{x}_{\mathrm{sm}}$. The figures show the
0.33 was measured. For composites containing c-NFC (light gray columns), the MFI increased with the fibril loading. This is an indication for degradation of the PLA matrix, leading to a reduction of the PLA chain length [19] and therefore to a lower viscosity. In contrast, the MFI of composites containing c-NFC-hex (medium gray columns) and c-NFC-hex ${ }_{s m}$ (dark gray columns) showed a faint decrease with higher fibril loadings, which indicates that a better fiber dispersion was obtained for these nanofibers, especially for the solvent mixed composites.

These results were confirmed by shear tests in parallel plate geometry. Figure 6 shows frequency scans of neat PLA and composite samples containing $7.5 \%$ of fibrils at $180{ }^{\circ} \mathrm{C}$ (left) and $200{ }^{\circ} \mathrm{C}$ (right), displaying the shear storage modulus G' (top), shear loss modulus G” (center) and the viscosity $\gamma$ (bottom) in dependence of the angular frequency $\omega$. At $180{ }^{\circ} \mathrm{C}, \mathrm{G}$ ' and $\mathrm{G}$ " of the neat PLA melt (green dots) increased over several decades with increasing
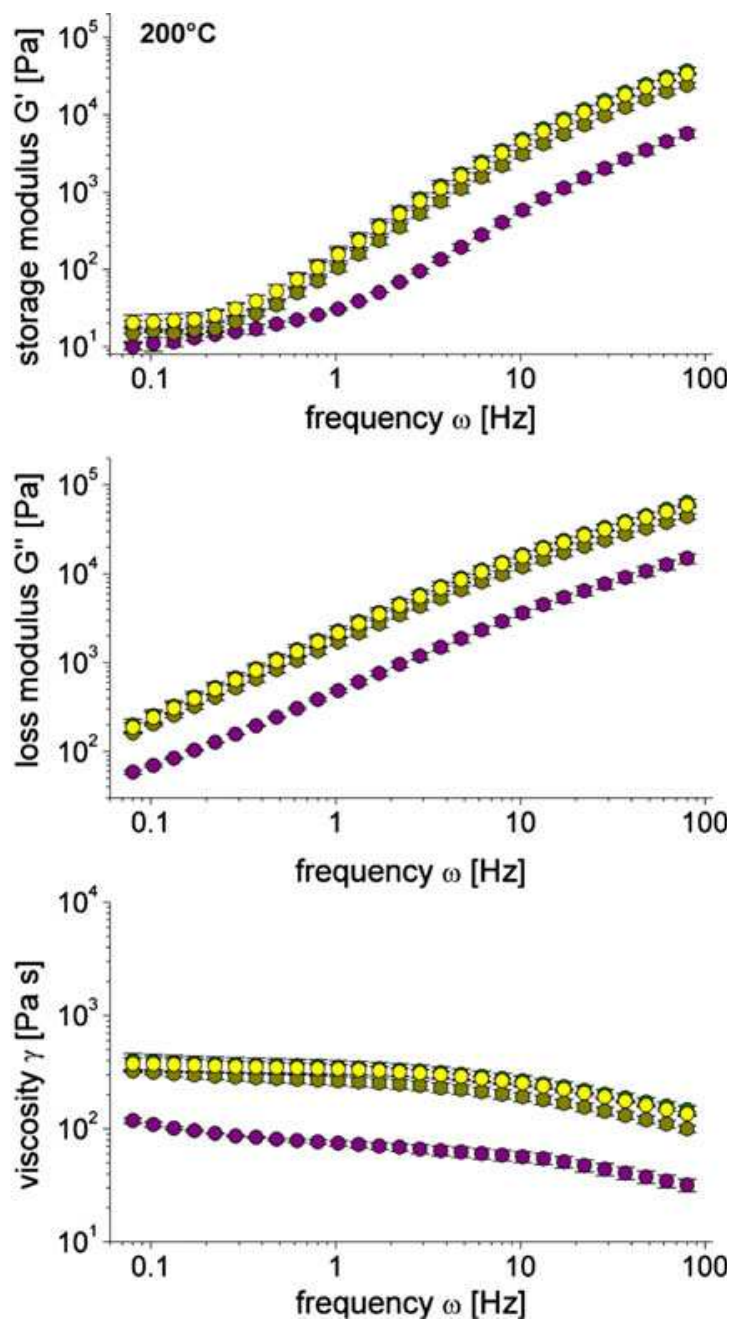

storage modulus G' (top), loss modulus G" (center) and viscosity $\gamma$ (bottom) in dependence of the frequency $\omega$ of the samples at $180{ }^{\circ} \mathrm{C}$ (left) and $200{ }^{\circ} \mathrm{C}$ (right) 

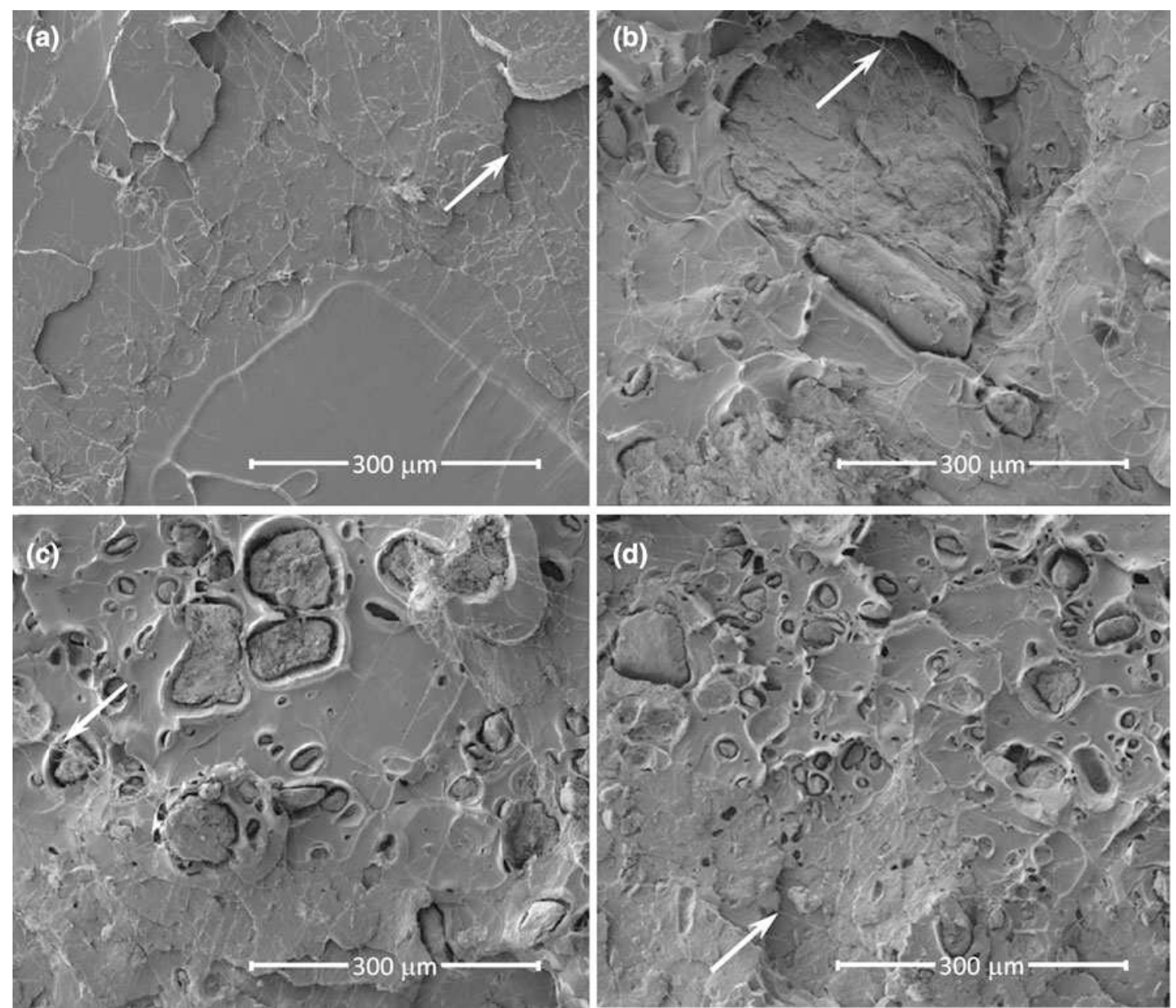

Fig. 7 Scanning electron microscopy (SEM) images of a neat PLA and composites containing PLA and $7.5 \% \mathrm{w} / \mathrm{w}$ of $\mathbf{b} \mathrm{c}-\mathrm{NFC}, \mathbf{c} \mathrm{c}-\mathrm{NFC}-$ hex and d c-NFC-hex sm. The images show fracture surfaces of

tensile testing specimens. Arrows indicate polymer fibrils. Magnifications are approximately $130 \times$

the frequency from 0.08 to $80 \mathrm{~Hz}$. The gel point $\left(\mathrm{G}^{\prime}=\mathrm{G}\right.$ ”) was not reached in the whole frequency range measured. The viscosity of the neat PLA melt was almost constant at $2 \mathrm{kPa}$ s for low frequencies and decreased with higher frequencies (shear thinning effect). Almost identical responses for G', G' and $\gamma$ were obtained for composites containing c-NFC-hex (olive dots) and c-NFC-hex sm $_{\text {(yel- }}$ low dots). Composites containing c-NFC (purple dots) showed the same behavior with clearly lower values. The same trends were observed at $200{ }^{\circ} \mathrm{C}$, with lower values for all samples measured.

The fractured surfaces of tensile test specimens of neat PLA (Fig. 7a) and composites containing $7.5 \% \mathrm{w} / \mathrm{w}$ of c-NFC (Fig. 7b), c-NFC-hex (Fig. 7c) and c-NFC-hex ${ }_{s m}$ (Fig. 7d) were analyzed using scanning electron microscopy. The fractured surface of neat PLA shows mainly brittle fracture, indicated by smooth surfaces. In addition, some very thin and long polymer fibrils span over the surface (indicated with arrows). These might origin from a plastic fracture mechanism, allowing the material to yield and form long PLA fibrils. When the fibrils finally break they seem to buckle and fall onto the fractured surface. The same kind of PLA polymer fibrils was also observed on the fractured surface images of the composites and they should not be confused with the cellulose fibrils synthesized with the aim to reinforce the polymer matrix. Clearly, all cellulose fibrils (c-NFC, c-NFC-hex and c-NFC-hex ${ }_{s m}$ ) showed excessive formation of low aspect ratio agglomerates. There is no evidence of a fibrillar network in either of the composites, even though the size of agglomerates clearly decreases in the order c-NFC (agglomerates with diameters in the range of approximately $300 \mu \mathrm{m}$ and more were found) $>$ c-NFC-hex (approximately $100 \mu \mathrm{m}$ and lower) $>$ c-NFC-hex $x_{s m}$ (approximately $50 \mu \mathrm{m}$ and lower). The images 
of c-NFC-hex (Fig. 7c) and c-NFC-hex ${ }_{s m}$ (Fig. 7d) also clearly show that the interaction/adhesion between the fibril agglomerates and the PLA is very poor.

In this study, a combination of several parameters might be the reason for the observed restricted reinforcement of PLA. First, the carboxymethylation of NFC decreased the crystallinity of the original nanofibers, and by that lowered the modulus of the fibrils. Second, the decreased strength of the composites with increased NFC contents indicated a poor interaction between the PLA and the cellulose nanofibers, modified or not. Third, a poor dispersion of the dried NFC powders inside the polymer matrix has been obtained during the extrusion. Apart from the poor fibermatrix interface mentioned previously, this problem may also be linked with the preparation of the materials prior to the extrusion. Fourth, the use of surface charged cellulose nanofibers may have negatively impacted the thermal stability of the composite materials. In addition to the color change of PLA-c-NFC samples, MFI measurements and measurements of viscoelastic properties in shear of the polymer melts showed that the thermal stability of PLA was reduced when compounded with c-NFC. A possible explanation for these results may come from the reaction of carboxylate groups of c-NFC with the PLA at elevated temperatures (temperatures up to $200{ }^{\circ} \mathrm{C}$ were used in the extruder), therefore decreasing the thermal stability of the matrix. Moreover, it has been reported earlier that the presence of carboxylate groups leads to a decrease in the thermal stability of cellulose [18, 20,21]. In addition, a degrading effect of $\mathrm{DMAc} / \mathrm{LiCl}$ at elevated temperatures of $80-125^{\circ} \mathrm{C}$ on cellulose was reported. The mechanism included the formation of keteniminium ions, inducing hydrolytic cleavage of the glycosidic bonds in cellulose [22]. This process was accompanied by a yellow coloration. Consequently in our study, a similar mechanism involving charged compounds might also be responsible for the limited stability of c-NFC.

Composites containing c-NFC-hex or c-NFC-hex ${ }_{s m}$ were less susceptible to thermal degradation. This result might be related with a possible increased thermal stability of the esterified nanofibers as compared with c-NFC, and/or with the "blocking" of c-NFC carboxylate groups which therefore decreased the probability of PLA degradation. However, a reinforcing effect in terms of an increase in $G^{\prime}$ or viscosity was not found for any of the composites.

\section{Conclusions}

The aim of this study was to examine if dried nanofibrillated cellulose (NFC) in powder form, with different polarities, can be dispersed in PLA using an extrusion process, and to further evaluate its capacity to reinforce the PLA. To achieve our goal, different functionalized nanofibers were compounded with PLA using an extrusion process. Carboxymethylated NFC (c-NFC) was esterified with 1-hexanol in presence of a sulfuric acid catalyzer to yield c-NFC hexanoate (c-NFC-hex). In addition, a solvent mix (c-NFC-hex ${ }_{s m}$ ) was prepared by mixing equal amounts of dissolved PLA and dispersed c-NFC-hex and precipitating the mixture in ice-cold isopropanol. Composites with different fibril contents were prepared by diluting the masterbatches of 10/90 (c-NFC/PLA, c-NFC-hex/PLA and c-NFC-hex $x_{s m} /$ PLA) with neat PLA to $2.5,5$ and $7.5 \%$ $\mathrm{w} / \mathrm{w}$. The products were pelletized and injection molded to dog-bone shaped tensile test samples and flat discs for dynamic mechanical analysis and melt shear measurements. The fractured surfaces of the tensile test samples were analyzed by scanning electron microscopy.

The results showed that the types of fibrils examined in this work did not disperse, distribute or form a network within the PLA matrix but exhibited instead extensive agglomeration. Also, poor interaction with the matrix was observed in all composites, as demonstrated by DMA and SEM. The poor interaction and agglomerations affected the strength of the composites negatively. In addition, the thermal degradation of the PLA matrix, due to a possible reaction between the nanofibers carboxylate groups and the ester chains in high temperature, might also be a reason for the lowered mechanical properties of the composites. Furthermore, a decrease in crystallinity of the cellulose fibrils due to carboxymethylation could also have lowered the stiffness of the cellulose fibrils, and by that their reinforcing capability.

Even though this study on esterification of c-NFC with 1-hexanol for extrusion with PLA did not result in composite materials with increased mechanical properties, it should remain the subject of further research to find suitable and simple strategies for the development of composite materials with three-dimensional networks of nanofibrillated cellulose.

Acknowledgments The authors express their thanks to Aji Mathew and Maiju Hietala for their help during extrusion and injection molding, and Christian Walder (EMPA) for his support concerning the melt shear tests. The State Secretariat for Education and Research (SER) is gratefully acknowledged for the financial support of this work.

\section{References}

1. Auras R, Harte B, Selke S (2004) Macromol Biosci 4:835-864

2. Garlotta D (2001) J Polym Environ 9:63-84

3. Siro I, Plackett D (2010) Cellulose 17:459-494

4. Samir M, Alloin F, Dufresne A (2005) Biomacromolecules 6:612-626

5. Chazeau L et al (1999) J Appl Polym Sci 71:1797-1808

6. Favier V, Chanzy H, Cavaille JY (1995) Macromolecules 28: 6365-6367 
7. Marchessault RH, Morehead FF, Walter NM (1959) Nature 184:632-633

8. Turbak AF, Snyder FW, Sandberg KR (1983) J Appl Polym Sci Symp 37:815-827

9. Oksman K et al (2006) Compos Sci Technol 66:2776-2784

10. Mathew AP et al (2006) In: Oksman K, Sain M (eds) Cellulose nanocomposites: processing, characterization, and properties. American Chemical Society, Washington, pp 114-131

11. Petersson L, Kvien I, Oksman K (2007) Compos Sci Technol 67: 2535-2544

12. Bondeson D, Oksman K (2007) Compos A Appl Sci Manuf 38: 2486-2492

13. Bondeson D, Oksman K (2007) Compos Interfaces 14:617-630

14. Iwatake A, Nogi M, Yano H (2008) Compos Sci Technol 68:2103-2106
15. Suryanegara L, Nakagaito AN, Yano H (2009) Compos Sci Technol 69:1187-1192

16. Tingaut P, Zimmermann T, Lopez-Suevos F (2010) Biomacromolecules 11:454-464

17. Jonoobi $\mathrm{M}$ et al (2010) Compos Sci Technol 70:1742-1747

18. Eyholzer C et al (2010) Cellulose 17:19-30

19. Lee K-Y, Blaker JJ, Bismarck A (2009) Compos Sci Technol 69: 2724-2733

20. Fukuzumi $\mathrm{H}$ et al (2009) Biomacromolecules 10:162-165

21. Leza ML et al (1989) Die Angewandte Makromolekulare Chemie 168:195-203

22. Rosenau $\mathrm{T}$ et al (2003) Polymer 44:6153-6158 